\title{
On the mesh resolution of industrial LES based on the DNS of flow over the T106C turbine
}

\author{
Mohammad Alhawwary and Z.J. Wang ${ }^{*}$ (D)
}

\author{
*Correspondence: zjw@ku.edu \\ ${ }^{1}$ Department of Aerospace \\ Engineering, University of Kansas,
} Lawrence, KS, 66045, US

\begin{abstract}
A high-order Navier-Stokes solver based on the flux reconstruction (FR) or the correction procedure via reconstruction (CPR) formulation is employed to perform a direct numerical simulation (DNS) and large eddy simulations (LES) of a well-known benchmark problem - transitional flow over the low-pressure $\mathbf{T 1 0 6 C}$ turbine cascade. Hp-refinement studies are carried out to assess the resolution requirement. A 4th order (p3) simulation on the fine mesh is performed with a DNS resolution to establish a "converged" solution, including the mean pressure and skin-friction distributions, and the power spectral density in the wake. Then LES on the coarse and fine meshes with lower order schemes are conducted to assess the mesh and order dependence of the solution. In particular, we study the error in the transition location, the mean skin-friction distribution, and the mean lift and drag coefficients. These h- and $\mathrm{p}$-refinement studies provide a much-needed guideline in $\mathrm{h}$ - and $\mathrm{p}$ - resolutions to achieve a certain level of accuracy for industrial LES applications.
\end{abstract}

Keywords: Direct numerical simulations, Large eddy simulations, Transitional flows, High-order FR/CPR, T106C, Skin-friction

AMS Subject Classification: 76F65; 76F06; 65M60; 65M70

\section{Introduction}

Large eddy simulation [1] has received increased attention for industrial applications over the past decade for challenging vortex-dominated turbulent flows [2-6]. Direct numerical simulations have also been used to study interesting flow physics at low Reynolds numbers, e.g. [7]. This is in part due to the advancements in computational algorithms and computing power of modern computers which paved the way for simulating more practical flow problems. In its 2030 vision [8], NASA has predicted that scale resolving simulations will be increasingly used for vortex-dominated turbulent flow simulations such as rotorcraft flows and turbomachinery flows in aircraft engines.

The Reynolds Averaged Navier-Stokes (RANS) approach, which models all turbulent scales, has been widely used in industrial applications and its efficiency and accuracy were shown for non-separated flows. However, the application of RANS to highly separated turbulent flow has not been very successful either because a statistically steady mean flow does not exist or the turbulence model is not adequate for such problems. In addition,

(C) The Author(s). 2019 Open Access This article is distributed under the terms of the Creative Commons Attribution 4.0 International License (http://creativecommons.org/licenses/by/4.0/), which permits unrestricted use, distribution, and reproduction in any medium, provided you give appropriate credit to the original author(s) and the source, provide a link to the Creative Commons license, and indicate if changes were made. 
RANS has serious limitations when it is used to tackle heat transfer and combustion problems $[9,10]$. On the other hand, DNS, which resolves all turbulent scales, is prohibitively expensive even for problems at moderate Reynolds numbers. LES lies between RANS and DNS in terms of both accuracy and cost. Thus it opens a new avenue for tackling real world problems with superior accuracy and manageable cost.

In particular, turbomachinery flow is arguably one of the most challenging problems in computational fluid dynamics (CFD). These types of problems involve complex geometries and physics that requires high fidelity simulations and high accuracy [11]. Applications of LES and DNS simulation in turbomachinery problems have been conducted by many researchers [12-18]. Most previous computations have utilized finite difference (FD), compact difference (CD) $[14,19]$, and finite-volume (FV) $[13,20,21]$ methods for the space discretization. While these methods possess desirable properties such as simplicity and ease of implementation, they suffer from either the lack of geometric flexibility in the case of (FD,CD) or larger stencils/less locality in the case of high-order (FV) schemes.

Therefore, a number of adaptive high-order methods capable of handling unstructured grids have been developed over the past few decades [22,23]. These methods offer higher than 2nd order accuracy in space in addition to the compact/local nature of the required stencils. Thus, these methods are well suited for modern computing architectures such as GPUs or hybrid CPU/GPU due to their inherent element-local structure. In addition, they can handle different types of unstructured grids and hence can be applied to a wide range of industrial applications in contrast to classical FD/CD methods. Recently, a unifying framework called flux reconstruction [24] that encompasses several adaptive high-order methods was introduced by Huynh for hyperbolic conservation laws. This method was further extended to hybrid unstructured meshes $[25,26]$. The class of adaptive high-order methods also includes the spectral difference method (SD) [27], spectral volume method (SV) [28], the discontinuous Galerkin method (DG) [29-31], and the hybrid DG/FV method [32].

In LES, there is the physical dissipation that is associated with the molecular viscosity. Numerical schemes introduce additional numerical dissipation in addition to the subgrid-scale (SGS) model dissipation. It has been shown that in some cases SGS models may be detrimental to the solution quality [33-36]. In practice, implicit LES (ILES) has proved its potential for turbulent flow simulations [4, 5, 37-39]. In the context of LES, high-order adaptive methods such as DG and FR/CPR have been shown to resolve a wider spectrum than the classical FD method, and are comparable to the CD method in terms of resolution power [40, 41].

In this paper, we employ an in-house FR/CPR solver called hpMusic for the compressible Navier-Stokes equations to conduct DNS and LES simulations of a wellknown benchmark problem from one of the International Workshops on HighOrder CFD Methods [42], flow over the low-pressure turbine blade T106C cascade [43]. We focus on assessing the resolution required for a given error in the friction coefficient (Cf), the energy spectrum, and mean lift and drag coefficients. Some estimates are provided about the grid resolution, (streamwise, normal, spanwise) directions in wall units, to achieve a certain level of accuracy. We hope to help the turbomachinery community conduct faster and reliable LES to aid the design process. 
The paper is organized as follows, Section 2 introduces the basic formulation of the FR/CPR method. We then describe the DNS of the T106C test case in Section 3. A study of LES resolution is presented in Section 4. Finally, conclusions are summarized in Section 5.

\section{The FR/CPR method}

The FR/CPR method introduced by Huynh [44] is a nodal numerical formulation for solving hyperbolic partial differential equations, which was extended to hybrid unstructured grids for conservation laws [25]. From the FR/CPR formulation, several interesting methods can be derived such as the SV, SD, and DG schemes among others [24, 25, 45-47]. In this large family of schemes, some schemes may provide larger time steps than the DG scheme [45]. Several groups have shown that the FR/CPR method is more efficient than the DG method [48]. For a complete review of the method, the reader can consult the following references $[23,49,50]$.

In this section we present the FR/CPR method for a hyperbolic conservation law

$$
\frac{\partial \mathbf{U}}{\partial t}+\nabla \cdot \mathbf{F}(\mathbf{U})=0
$$

with initial and boundary conditions, where the vector $\mathbf{U}$ consists of the conservative variables, and $\mathbf{F}$ is the flux. In the FR/CPR framework, the computational $\mathcal{D}$ domain is discretized into a set of $N_{e}$ non-overlapping elements $\Omega_{i}$ such that $\mathcal{D}=\cup_{i=1}^{N_{e}} \Omega_{i}$. By introducing an arbitrary test function $W$ in each $\Omega_{i}$, the weighted residual formulation of Eq. 1 on $\Omega_{i}$ can be expressed as

$$
\int_{\Omega_{i}}\left[\frac{\partial \mathbf{U}}{\partial t}+\nabla \cdot \mathbf{F}(\mathbf{U})\right] W \mathrm{~d} \Omega=0 .
$$

We approximate the conservative variables $\mathbf{U}_{i}$ inside each element by degree $p$ polynomials using their nodal values at a set of points called solution points (SPs). After applying integration by parts to the divergence of flux, replacing the normal flux term with a common Riemann flux $F_{c o m}^{n}$ and performing integration by parts again, we obtain

$$
\int_{\Omega_{i}} \frac{\partial \mathbf{U}_{i}}{\partial t} W \mathrm{~d} \Omega+\int_{\Omega_{i}} W \nabla \cdot \mathbf{F}\left(\mathbf{U}_{i}\right) \mathrm{d} \Omega+\int_{\partial \Omega_{i}} W\left[F_{c o m}^{n}-F^{n}\left(\mathbf{U}_{i}\right)\right] \mathrm{d} S=0 .
$$

The common Reimann flux $F_{c o m}^{n}$ is computed using a Riemann solver

$$
F_{c o m}^{n}=F_{c o m}^{n}\left(\mathbf{U}_{i}, \mathbf{U}_{i+}, \mathbf{n}\right)
$$

where $\mathbf{U}_{i+}$ denotes the solution outside the current element, and $\mathbf{n}$ is the outward normal vector of the interface under-consideration. The normal flux at the interface is defined as

$$
F^{n}\left(\mathbf{U}_{i}\right)=\mathbf{F}\left(\mathbf{U}_{i}\right) \cdot \mathbf{n} .
$$

In order to eliminate the test function $W$, the face integral in Eq. 3 is transformed into an element integral. In order to achieve that, a "correction feild" $\delta_{i}$ is defined in each element as

$$
\int_{\Omega_{i}} W \delta_{i} \mathrm{~d} \Omega=\int_{\partial \Omega_{i}} W \llbracket F^{n} \rrbracket \mathrm{d} S
$$


where $\left[F^{n} \rrbracket=F_{c o m}^{n}-F^{n}\left(\mathbf{U}_{i}\right)\right.$ is the normal flux jump. Combining Eqs. 3 and 6 we get

$$
\int_{\Omega_{i}}\left[\frac{\partial \mathbf{U}_{i}}{\partial t}+\nabla \cdot \mathbf{F}\left(\mathbf{U}_{i}\right)+\delta_{i}\right] W \mathrm{~d} \Omega=0 .
$$

After some manipulations and by projecting the divergence of flux term onto the space of degree $p$ polynomials [25], the final formulation for each solution point $j$ is

$$
\frac{\partial \mathbf{U}_{i, j}}{\partial t}+\Pi_{j}\left[\nabla \cdot \mathbf{F}\left(\mathbf{U}_{i}\right)\right]+\delta_{i, j}=0
$$

where $\Pi_{j}$ is the projection operator and subscript $j$ denotes the $j$-th solution point in the element.

Discretization of the viscous fluxes including the gradient of the conservative variables follows the second approach of Bassi and Rebay (BR2) [24, 46, 51]. After applying the space discretization, Eq. 8 reduces to a nonlinear ordinary differential equation (ODE) of the form

$$
\frac{\partial \mathbf{U}}{\partial t}=\mathbf{R}(\mathbf{U})
$$

where $\mathbf{R}$ denotes the residual of the equation. The time marching is achieved using the implicit 2nd order backward difference formula (BDF2) coupled with a nonlinear block LU-SGS (BLU-SGS) [52, 53] solver.

\section{DNS of transitional flow over the T106C blade}

\subsection{Case definition}

The T106C case is selected as a challenging transitional flow problem for CFD simulations and has been widely used in assessing both numerical discretization accuracy and studying turbulent flow physics associated with such types of flows $[12,13,16,21,54-56]$. The configuration of the considered problem follows the setup introduced in the 4th International Workshop on High-Order CFD Methods [42]. The blade has a chord of $C=0.093 \mathrm{~m}$, a pitch to chord ratio of 0.95 , and a span to chord ratio of $10 \%$. The inlet condition for the cascade is chosen such that the isentropic exit Mach number is $M_{i s}=0.65$ and the Reynolds number is $R e_{i s}=80,000$ based on the isentropic exit velocity. The inlet flow angle for this case is $32.7^{\circ}$. This condition results in a transitional flow which is characterized by a long laminar separation bubble on the suction surface that transitions to turbulence further downstream leading to a fully turbulent wake.

The computational domain consists of six outer boundaries in addition to the blade wall, see Fig. 1. The imposed boundary conditions are periodic for the top and bottom boundaries and two span-wise boundaries, and characteristic inflow condition ( specifying inlet total pressure and temperature and flow angle) for the inlet boundary, and characteristic outflow condition (imposing the outlet static pressure) at the outlet boundary. The wall boundary is specified as a no-slip adiabatic boundary condition. The isentropic Mach number $\left(M_{i s}\right)$ on the blade surface is defined as

$$
M_{i s}=\sqrt{\frac{2}{\gamma-1}\left(\left(\frac{P_{\infty}}{P_{2 s}}\right)^{(\gamma-1) / \gamma}-1\right)},
$$

where $P_{2 s}$ is the pressure on the blade surface, and $P_{\infty}$ is the exit pressure. 


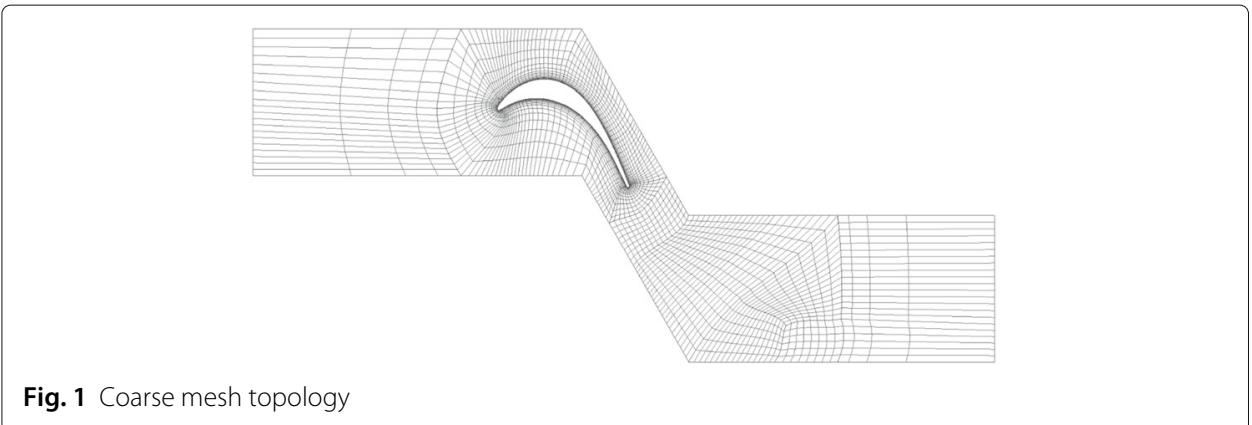

\section{2 $\mathrm{H}$ - and p-refinement studies}

For the purpose of establishing a DNS solution, both grid ( h-refinement) and order ( $\mathrm{p}$ refinement) convergence studies were preformed. We use two meshes (coarse and fine) and polynomial orders up to $p=4$. The coarse mesh consists of 18,588 degree 2 hexahedral elements and the fine mesh is obtained from the coarse one by uniform refinement in each direction resulting in 148,704 elements Fig. 2. The coarse mesh is extruded from a 2D mesh with 6 layers in the spanwise direction. In order to have matching periodic edges at the top and bottom boundaries, we designed a sophisticated block-structured mesh topology as shown in Fig. 1, which is generated using the PointWise mesh generation software. The mesh topology consists of an O-type block around the blade surface for resolution control in the boundary layer. Outside this $\mathrm{O}$-grid region a series of $\mathrm{H}$-type blocks are used. The grid growth rate in the wall normal direction inside the O-grid boundary layer block is 1.35 . Outside the boundary layer region the mesh size grows gradually with a ratio that is less than 1.5 in most regions. It is worth noting that although the grid is of a block-structured type, our numerical solver and scheme deal with it as an unstructured grid topology. The use of a block-structured type was only to allow better control of the grid coarsening for the LES study in the streamwise, spanwise, and normal directions.

In this study we always start from a $p 0$ steady solution and then restart to the $p 1$ unsteady simulation and we continue to restart as the order increases. This is done after each simulation converges in time with respect to the mean flow quantities. We define the characteristic time as $T_{c}=C / U_{\infty}$. The time averaging starts after the flow reaches a statistically steady state which based on our tests is about $\approx 108 T_{c}$. This was identified by monitoring the time histories of lift and drag, making sure it reaches that statistically steady state. It is hard for these types of flows to determine the correct transient region due to the highly unsteady nature. We note that not all the cases need this time to reach a statistically steady state but we are being more conservative here since some high-order

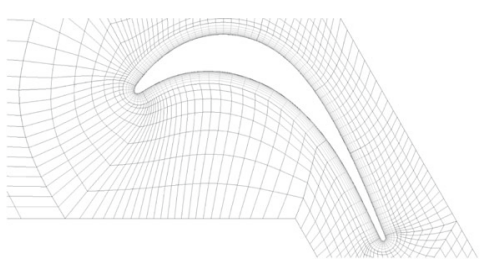

(a)

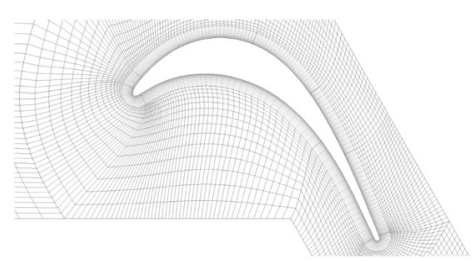

(b)

Fig. 2 Mesh quality near the blade. a Coarse mesh. b Fine mesh 
simulations needed this long. The time used for averaging the flow quantities is $216 T_{c}$. This ensures the convergence of these mean quantities after performing a spane-wise averaging as well. We also note that the time needed to converge the pressure distribution over the blade and hence the isentropic Mach number is much less than the time needed to converge the skin-friction.

The h- and p-refinement studies for the mean lift $\overline{C_{L}}$ and drag $\overline{C_{D}}$ coefficients are shown in Fig. 3. From this figure it can be clearly seen that the p3 ( $4^{\text {th }}$ order) solution on the fine mesh and $\mathrm{p} 4$ ( $5^{\text {th }}$ order) solution on the coarse mesh are nearly identical indicating the convergence of these quantities. In this paper, we study the mesh resolution in terms of the $X_{+}, Y_{+}, Z_{+}$, i.e., (streamwise, normal, spanwise) mesh resolutions in wall units. We define the equivalent $\widetilde{X}_{+}, \widetilde{Y}_{+}, \widetilde{Z}_{+}$for high-order multi-degree of freedom methods as

$$
\tilde{r}_{+}=r_{+} /(p+1), \quad r \in\{X, Y, Z\} .
$$

and the number of degrees of freedom (nDOF) per equation as

$$
\mathrm{nDOF}=N_{e} \times(p+1)^{d},
$$

where $N_{e}$ is the number of elements in the mesh, and $d=3$ is the dimension of the problem.

Table 1 shows the simulation data for all the cases including the averaged $\widetilde{X}_{+}, \widetilde{Y}_{+}, \widetilde{Z}_{+}$on the blade wall, based on wall normal units. From this table we can see that the p3 solution on the fine mesh has the highest resolution in terms of averaged $\left(\widetilde{X}_{+}, \widetilde{Y}_{+}, \widetilde{Z}_{+}\right)$values. The distribution of $\widetilde{X}_{+}, \widetilde{Y}_{+}, \widetilde{Z}_{+}$is also presented in Fig. 4 for the $\mathrm{p} 3$ on the fine mesh. It can be seen clearly from this figure that this $\mathrm{p} 3$ fine mesh resolution is a typical DNS resolution for such type of flow $[12,14]$.

Next, we consider the distribution of the pressure coefficient and the coefficient of streamwise skin-friction on the blade surface in Figs. 5 and 6. From these figures we can see that, similar to the mean $\overline{C_{L}}$ and $\overline{C_{D}}$ quantities, the $\mathrm{p} 3$ solution on the fine mesh is very close to the $\mathrm{p} 4$ solution on the coarse mesh. A closer look at these distribution is shown by zooming on the trailing edge in Fig. $5 \mathrm{~b}$ for the pressure coefficient $C_{p}$ and in Fig. $6 \mathrm{~b}$ for the coefficient of streamwise friction $C_{f s}$. In particular, for the $C_{f s}$ distribution we can see that the separation point is predicted correctly as in Hillewaert et al. [12] to be close to $0.7 C_{\text {axial }}$. The convergence in this transition region for the friction coefficient is much harder than the pressure coefficient, $\overline{C_{L}}$ and $\overline{C_{D}}$. This is due to the high intermittency of the separation and transition zones [12].

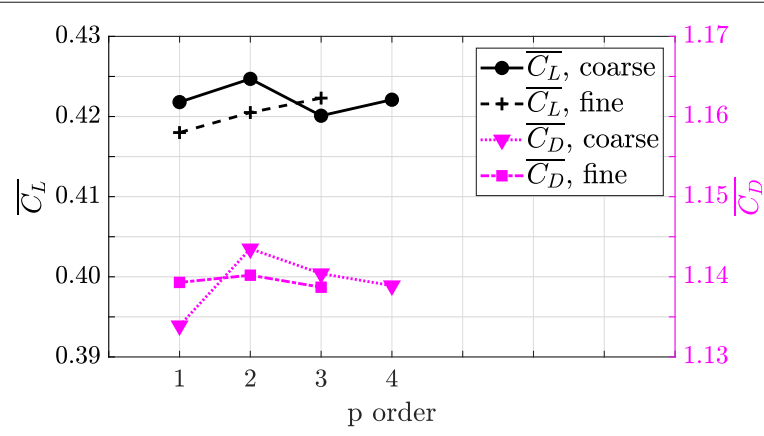

Fig. 3 Convergence of the mean lift $\overline{C_{L}}$ and drag $\overline{C_{D}}$ coefficients 
Table 1 Simulation data including, average $\left(\widetilde{X}_{+}, \widetilde{Y}_{+}, \widetilde{Z}_{+}\right)$over the blade surface, nDOF, and the mean $\overline{C_{L}}$ and $\overline{C_{D}}$ coefficients

\begin{tabular}{lllllll}
\hline Cases & $\tilde{X}_{+}$ & $\tilde{Y}_{+}$ & $\tilde{Z}_{+}$ & $n D O F \times 10^{6}$ & $\overline{C_{L}}$ & $\overline{C_{D}}$ \\
\hline p1 coarse & 31 & 1.44 & 34 & 0.1 & 0.4218 & 1.1339 \\
p2 coarse & 20 & 0.96 & 22 & 0.5 & 0.4247 & 1.1435 \\
p3 coarse & 16 & 0.74 & 17 & 1.2 & 0.4201 & 1.1404 \\
p4 coarse & 13 & 0.60 & 14 & 2.3 & 0.4221 & 1.1389 \\
p1 fine & 16 & 0.78 & 18 & 1.2 & 0.4180 & 1.1393 \\
p2 fine & 10 & 0.48 & 11 & 4.0 & 0.4205 & 1.1402 \\
p3 fine & 8 & 0.36 & 8 & 9.5 & 0.4223 & 1.1387 \\
\hline
\end{tabular}

The power spectral density (PSD) of the pressure and the velocity magnitude is computed at two points in the wake. The first point is near the T.E. with coordinates $(0.8591,-0.5137) C$, while the second one is further in the wake downstream of the blade at location $(0.9,-0.6) C$. For these points we conduct a spanwise average using four locations in the spanwise direction, $Z / C \in\{0.0,0.025,0.05,0.075\}$. The Welch's method of averaged periodograms [57] is utilized for the computation of the PSD. An efficient implementation of this method [58] is used in this paper. In this method the total time signal of the pressure is divided into subsets with $50 \%$ overlap between them and each subset is Hann windowed after the mean is subtracted. The definition of the PSD used in this work for any quantity of interest $G$ is

$$
\operatorname{PSD}(S t)=\frac{2(\hat{G})^{2}}{\Delta f C / U_{\infty}}=\frac{2(\hat{G})^{2}}{\Delta S t}
$$

where $\hat{G}$ is the one-sided amplitude as determined by performing a Fourier transform of the time signal, $S t=f C / U_{\infty}$ is the Strouhal number based on the chord $C$ and the exit velocity $U_{\infty}$, and $f$ is the frequency in $\mathrm{Hz}$. Note that there is additional scaling applied to $\hat{G}$ to account for multiplying by a window function [57]. The total time history used to compute the PSD is the same as the time needed for averaging the mean quantities of $216 T_{c}$. The windowed subsets are of length $\approx 35 T_{c}$ each, resulting in a total of 11 subsets, and the data are collected with $\Delta t=2.16 \times 10^{-3} T_{c}$. This results in a Strouhal number

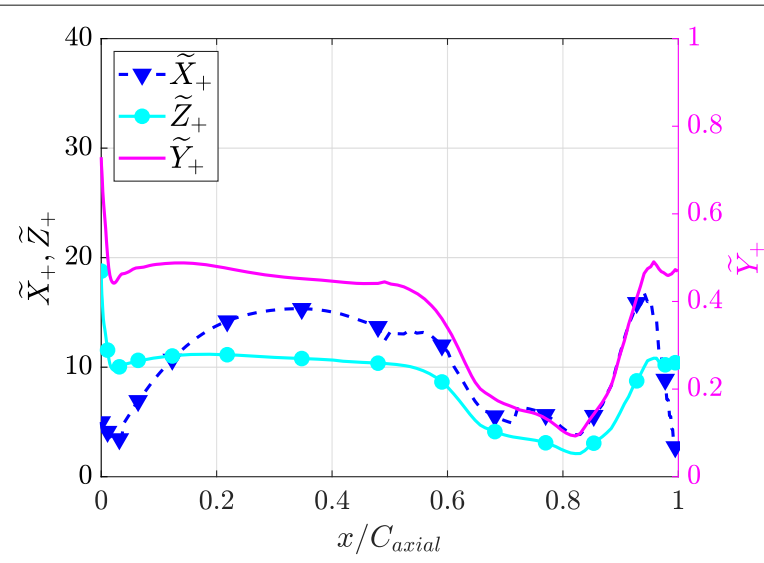

Fig. 4 Mesh normalized resolutions (based on wall units) on the suction side of the blade surface using p3 solution on the fine mesh (DNS) 


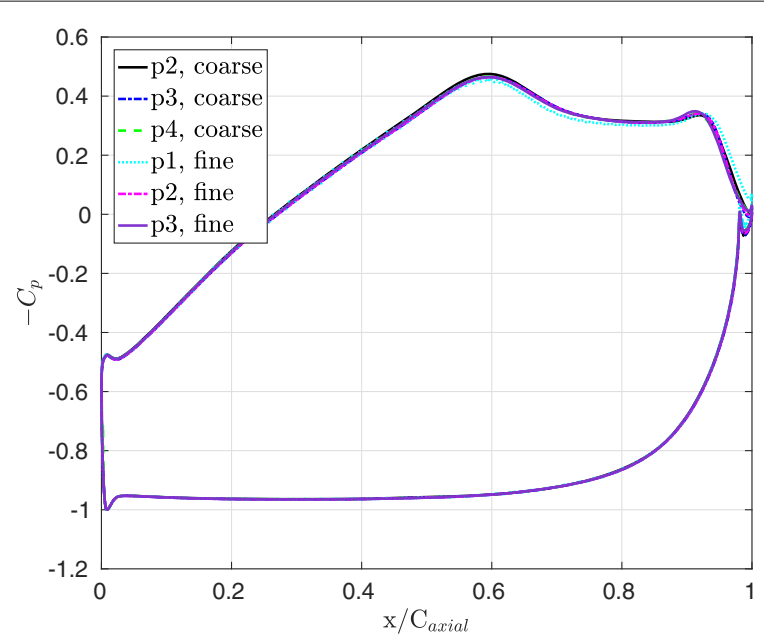

(a)

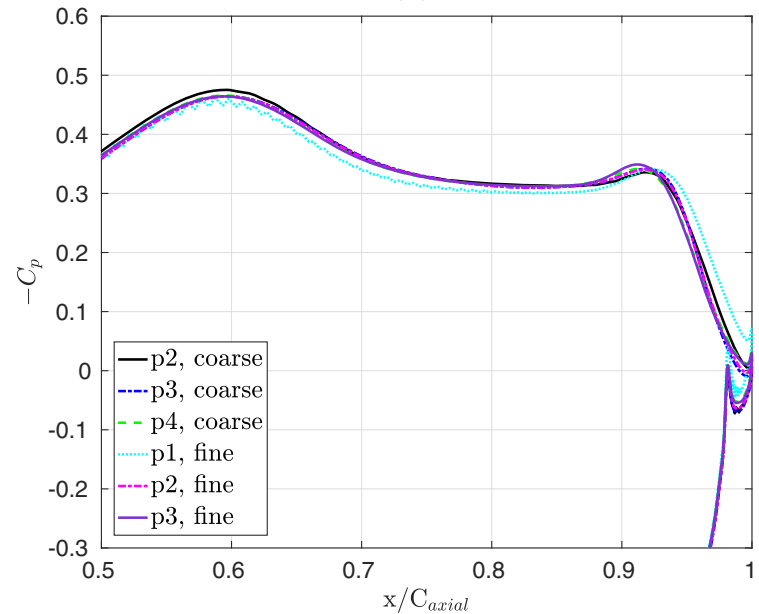

(b)

Fig. 5 Comparison of the time- and spanwise-averaged coefficient of pressure for all meshes and polynomial orders. (a) global view over the blade (b) enlarged view near the trailing edge

resolution of $\Delta S t \approx 0.03$ and a cutoff $S t_{\text {cutoff }} \approx 231$. In this work we have used the same spectral parameters for the computation of the PSD for all the cases.

Figure 7 presents the PSD of the pressure signal at wake point(2) after spanwise averaging the pressure at the four spanwise locations mentioned earlier. From this figure we can also see that all the cases were able to capture the positions of the peaks except the coarse mesh $\mathrm{p} 1$ results which missed the second peak, and the coarse mesh p2 which underestimated the PSD at the first peak. In addition, the pressure spectra in this figure follows the theoretical $-7 / 3$ slope based on the dimensional analysis of Kolmogorv's theory for isotropic turbulence [59-61]. We note that this slope is not completely agreed on in the literature but can be seen in some cases [62] and has been reported by Marty et al. [13] for the flow around the T106C case. In Fig. 8, the PSD of the velocity magnitude also showed a good agreement with the theoretical slope of $-5 / 3$. However, for the velocity spectrum the point near the T.E. showed a better trend than the one further in the wake. This spectra PSD study shows the consistent convergence of the mesh and order resolutions up to 


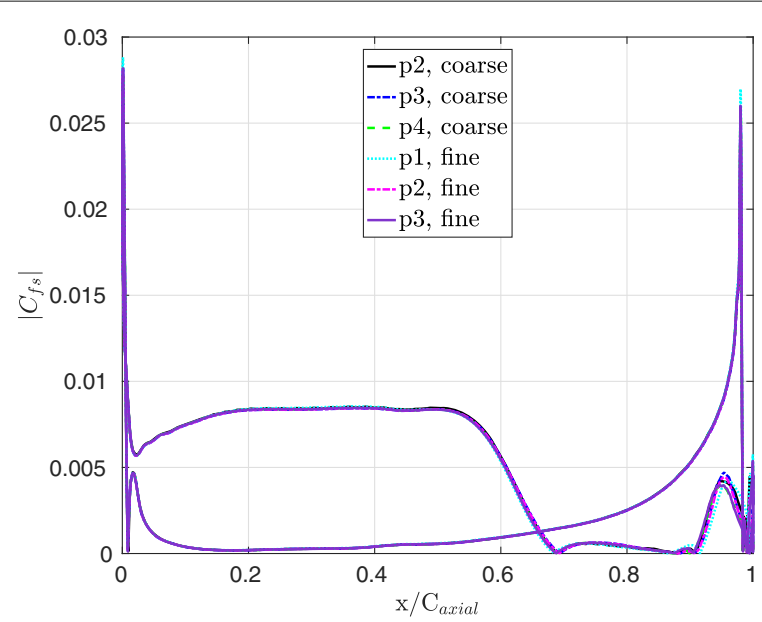

(a)

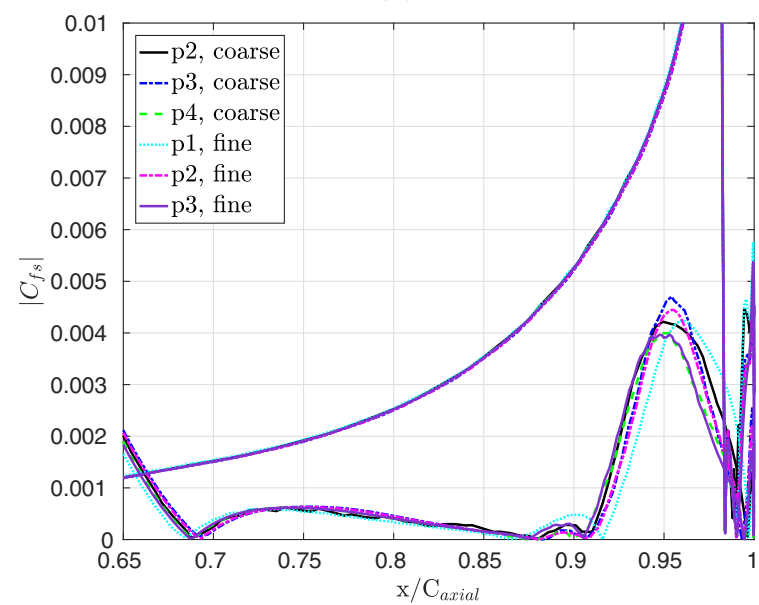

(b)

Fig. 6 Comparison of the time- and spanwise-averaged coefficient of the streamwise friction $C_{f s}$ for all meshes and polynomial orders. (a) global view over the blade (b) enlarged view near the trailing edge

the highest resolution of $\mathrm{p} 3$ on the fine mesh. Based on these results, we establish the $\mathrm{p} 3$ solution on the fine mesh as the DNS solution for the rest of the paper.

The comparison with experimental data of Michálek et al. [43] was rather difficult to obtain in the literature $[12,54,55]$. In Fig. 9, we present the comparison of the isentropic Mach number distribution on the blade surface with some of the results in the literature. From this figure it can be seen that on the pressure side we are able to show very good agreement with the experiment as in Hillewaert et al. [12]. However, on the suction side, there is a slight underprediction behavior up to the separation region and the suction peaks are overestimated. This observation is similar to most of the results in the literature and has been attributed to the difference in the inlet conditions between the experiment and the numerical simulations as conjectured by Hillewaert et al. [12]. We also note that Hillewaert et al. [12] has used a spanwise extent of $0.2 C$ and Garai et al. [16] has used a $0.24 C$ whereas our simulation only uses a $0.1 C$ spanwise extent. In the experiment the spanwise extent was $2.4 C$. The reason for the shorter spans in the numerical simulations 


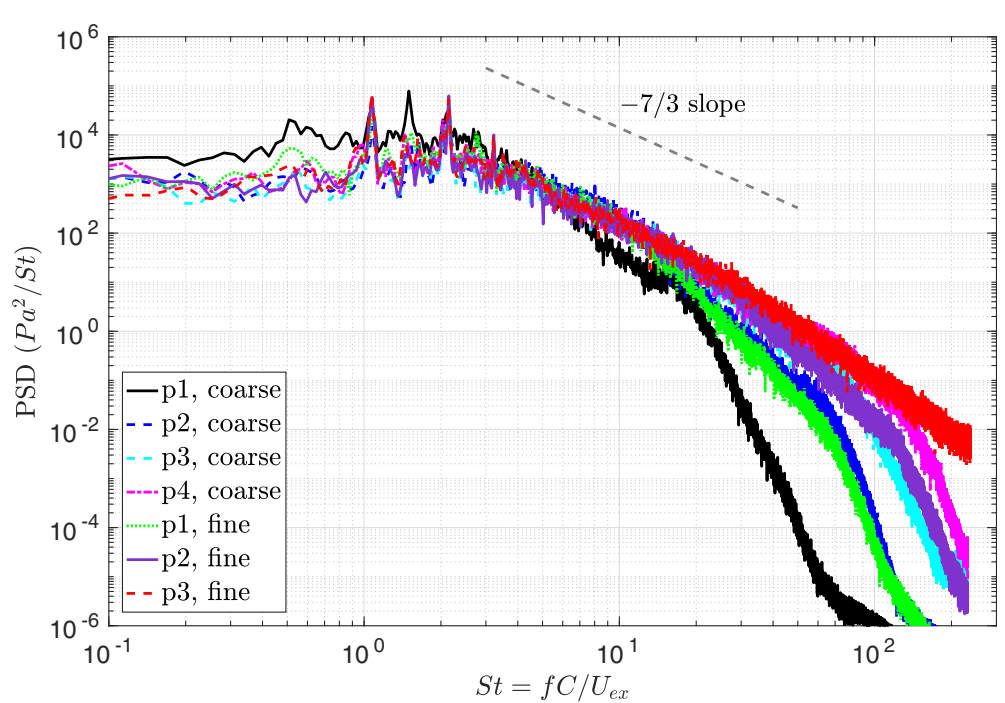

Fig. 7 Comparison of the PSD of pressure for all cases at wake point(2)

is to reduce the cost in addition to following the problem definition according to the highorder CFD workshop in our case. Moreover, in the previous numerical studies a grid or order dependence convergence was not systematically done to ensure reaching a DNS resolution. Nevertheless, we can see that our results are very close to Hillewaert et al. [12] who has reported having a $\widetilde{Y}_{+}<1.7$ over the blade surface.

Finally, a qualitative comparison between the DNS solution and one of the LES simulations ( $\mathrm{p} 2$ on the coarse mesh ) is shown in Fig. 10. In this figure we present the isosurfaces of the q-criterion colored by the axial velocity. This figure shows the small structures that are captured by the DNS resolution in comparison to the larger structures that the LES was able to capture. In particular, the LES resolution captures the coherent structures and their breakdown near the separation bubble and the vortex shedding phenomena in the wake. However, in the DNS case those coherent structures are less spanwise-periodic and straight than the LES case. The separation bubble extent is well captured by both resolutions as was evident for the distribution of $C_{f s}$ Fig. $6 \mathrm{~b}$.

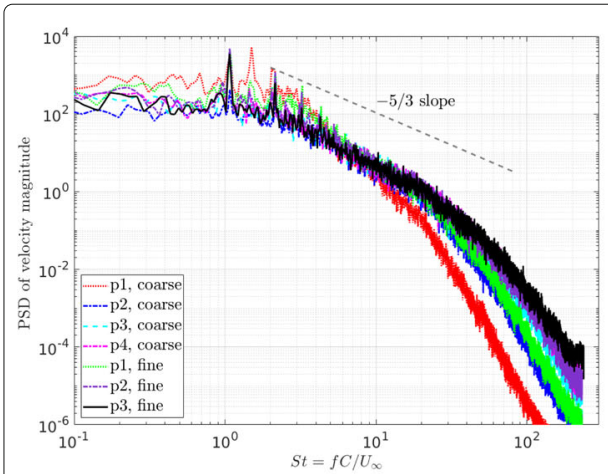

(a)

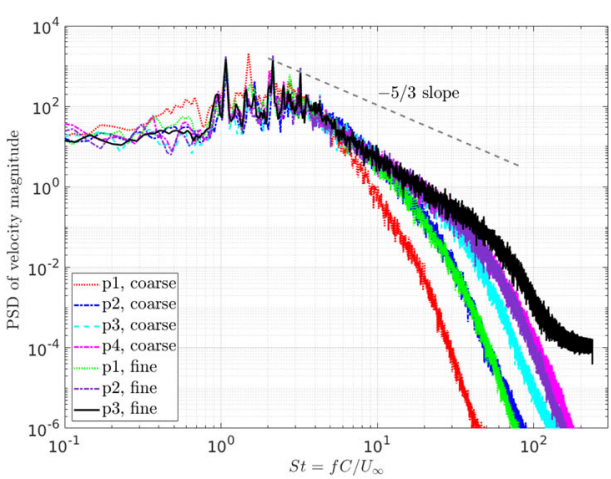

(b)

Fig. 8 Comparison of the PSD of the velocity magnitude for all cases at a wake point(1), b wake point(2) 


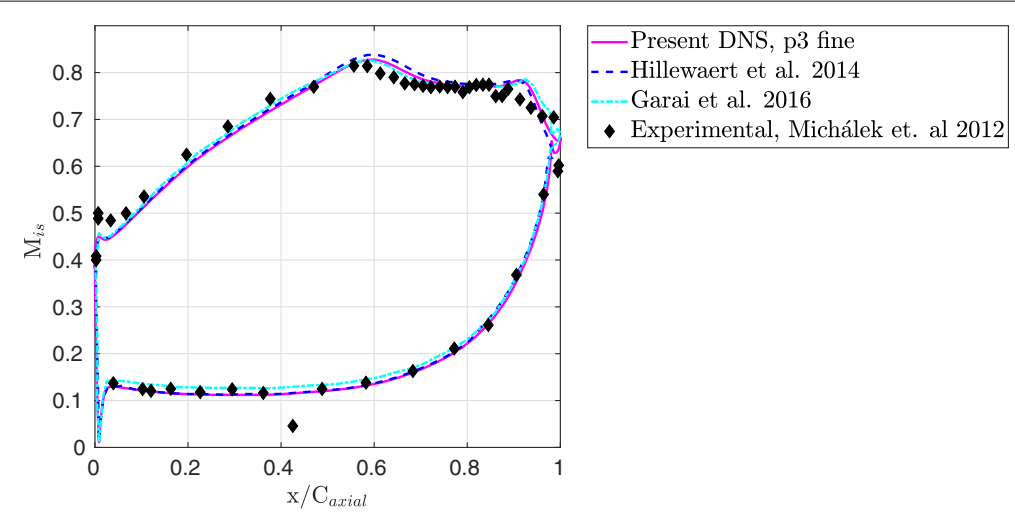

Fig. 9 Comparison of the time- and spanwise-averaged isentropic Mach number $M_{i s}$ with reference data

\section{LES resolution study}

In this section we study the effects of coarsening the mesh, in all directions, on the mean skin-friction distribution as well as the mean lift and drag coefficients. The goal is to provide a guideline for industrial simulations on the required resolutions for a given error tolerance. The choice of the block-structured mesh topology in Fig. 1 was made to facilitate this purpose. This ensures all the meshes are from the family.

We define our base LES resolution to be that of the $\mathrm{p} 2$ solution on the coarse mesh and denote the equivalent wall units of this setting by $\left(\widetilde{X}_{+}^{*}, \widetilde{Y}_{+}^{*}, \widetilde{Z}_{+}^{*}\right)=(20,0.96,22)$ with $0.5 M$ DOFs. We then coarsen the resolution in the streamwise to $2 \widetilde{X}_{+}^{*}$, normal to $2 \widetilde{Y}_{+}^{*}$, and spanwise to $2 \widetilde{Z}_{+}^{*}$ directions, one direction at a time to study the influence. In the spanwise direction, we also study the $3 \widetilde{Z}_{+}^{*}$ and $6 \widetilde{Z}_{+}^{*}$ resolutions where the latter consists of only one layer in the spanwise direction.

The distribution of the mean skin-friction coefficient $C_{f_{s}}$ on the blade surface is shown in Fig. 11 for the considered resolutions. From this figure we can see that the $2 \widetilde{X}_{+}^{*}, 2 \widetilde{Y}_{+}^{*}$, and $6 \widetilde{Z}_{+}^{*}$ resolutions failed to capture the first separation location and the separation bubble extent accurately. In particular, separation is delayed using these resolutions. On the other hand, the $2 \widetilde{Z}_{+}^{*}$ and $3 \widetilde{Z}_{+}^{*}$ resolutions accurately predicted the separation point while overestimating the length of the secondary bubble around $x=0.9 C_{\text {axial }}$. In addition, these two resolutions captured the same peak value in the turbulent region near the trailing edge (T.E.) of the blade $\left(x=0.95 C_{\text {axial }}\right)$ but shifted slightly with respect to the DNS

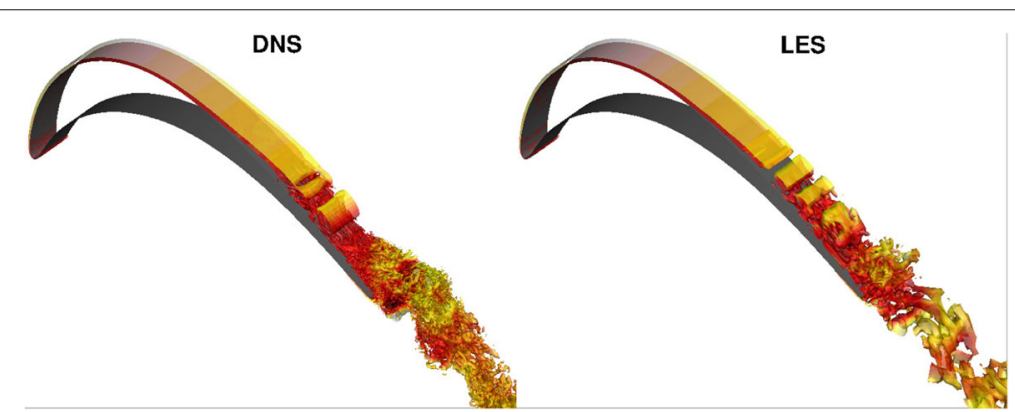

Fig. 10 Instantaneous Q-criterion contours colored by the axial velocity. The DNS solution consists of 9.5M DOF using a p3 discretization on the fine mesh whereas the LES consists of 0.5M DOF using a p2

discretization on the coarse mesh 


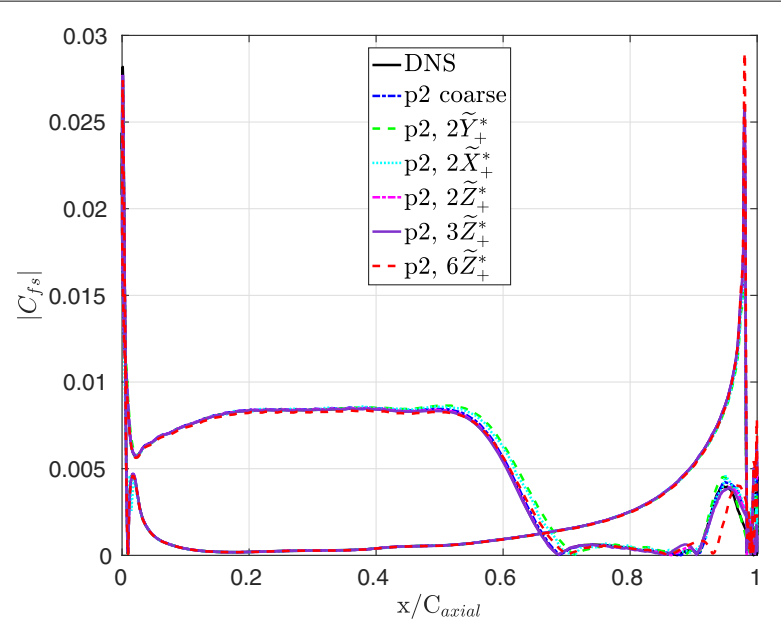

(a)

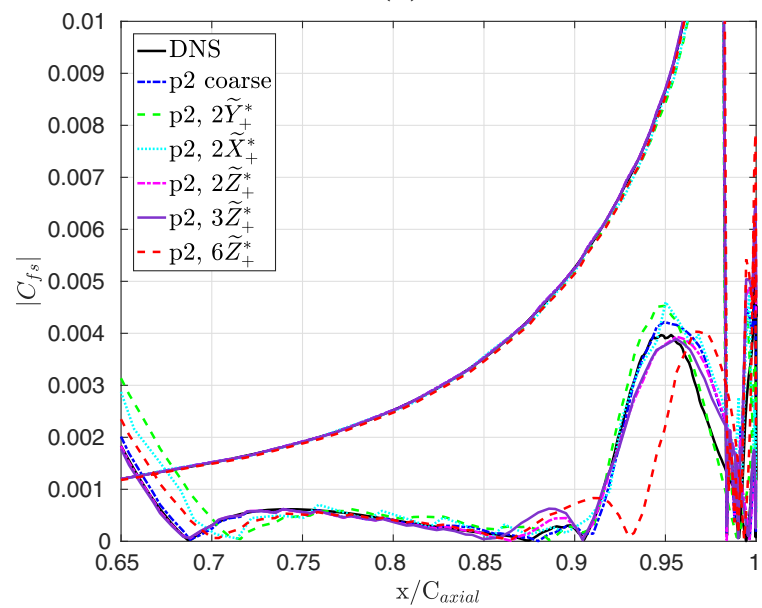

(b)

Fig. 11 Comparison of the time- and spanwise-averaged coefficient of streamwise friction for coarse LES simulations. Note that the starred resolution $\left(\widetilde{X}_{+}^{*}, \widetilde{Y}_{+}^{*}, \widetilde{Z}_{+}^{*}\right)=(20,0.96,22)$ corresponds to the $p 2$ coarse case. (a) global view over the blade (b) enlarged view near the trailing edge

results. The distribution of the mean pressure coefficient on the blade surface is presented in Fig. 12. From this figure we can see that indeed the $2 \widetilde{X}_{+}^{*}, 2 \widetilde{Y}_{+}^{*}$, and $6 \widetilde{Z}_{+}^{*}$ are inaccurate by a significant difference from both the DNS and the base p2 LES solutions. The other two cases, $2 \widetilde{Z}_{+}^{*}$ and $3 \widetilde{Z}_{+}^{*}$, are closer to the DNS and the base LES solutions. All cases showed very good accuracy on the pressure side except near the T.E. which indicates that high resolution is not needed for the pressure side due to the laminar attached boundary layer.

To take a closer look at the effects of these coarser resolutions, we compute the relative difference in the pressure and skin-friction coefficients with respect to the base $\mathrm{p} 2$ LES simulation, see Fig. 13. From this figure we can see that the difference in $C_{f s}$ for all the cases exceeded the $5 \%$ threshold in the turbulent region near the T.E. of the blade. On the pressure side of the blade, the difference is less than $5 \%$ for all cases with the $2 \widetilde{Z}_{+}^{*}$ resolution having the lowest difference. On the other hand, the differences in the $C_{p}$ distribution for all the cases are less than $5 \%$ except at some peaks which correspond to $C_{p}$ approaching zero. These peaks are the consequence of division by a small number. 


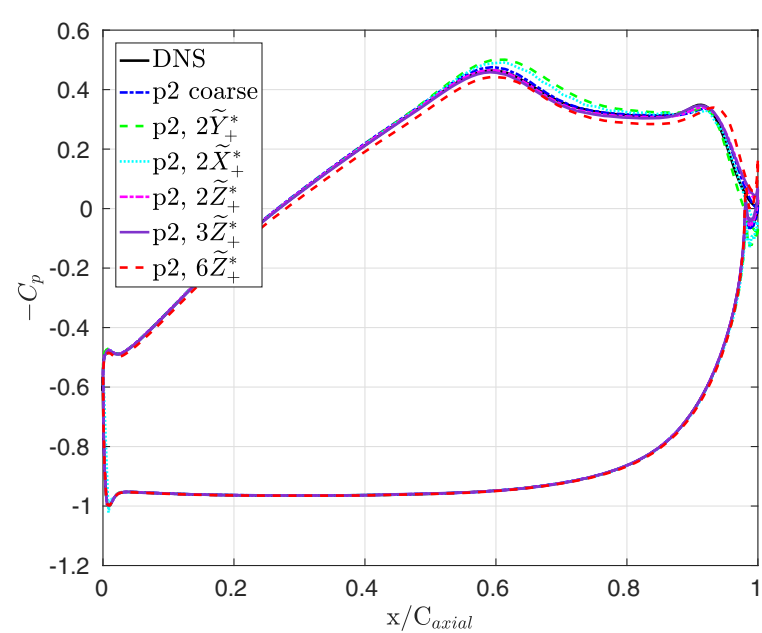

(a)

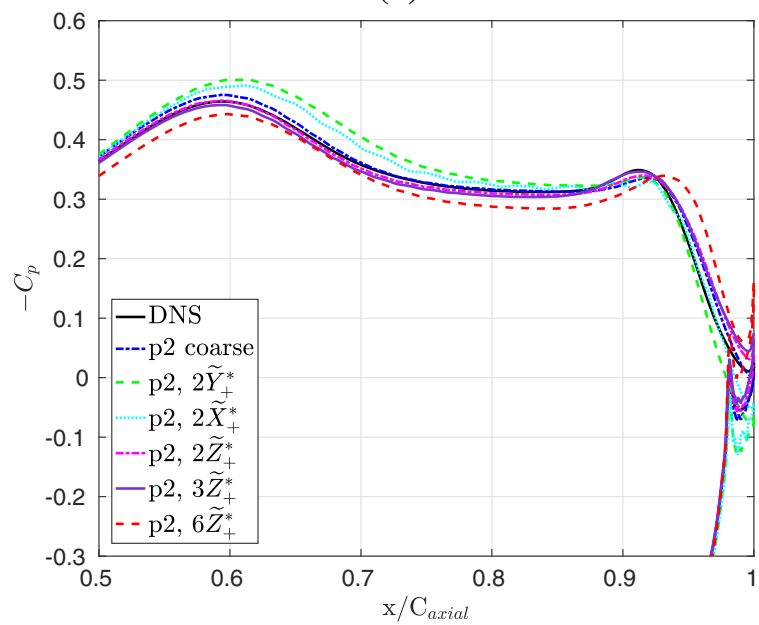

(b)

Fig. 12 Comparison of the time- and spanwise-averaged coefficient of pressure for coarse LES simulations. Note that the starred resolution $\left(\widetilde{X}_{+}^{*}, \widetilde{Y}_{+}^{*}, \widetilde{Z}_{+}^{*}\right)=(20,0.96,22)$ corresponds to the p2 coarse case. (a) global view over the blade $(\mathbf{b})$ enlarged view near the trailing edge

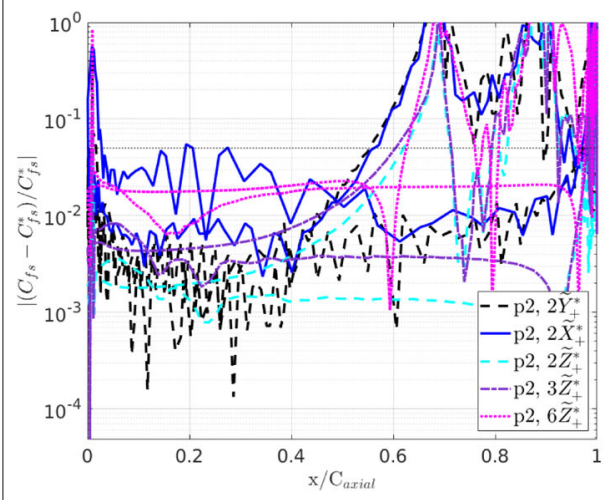

(a)

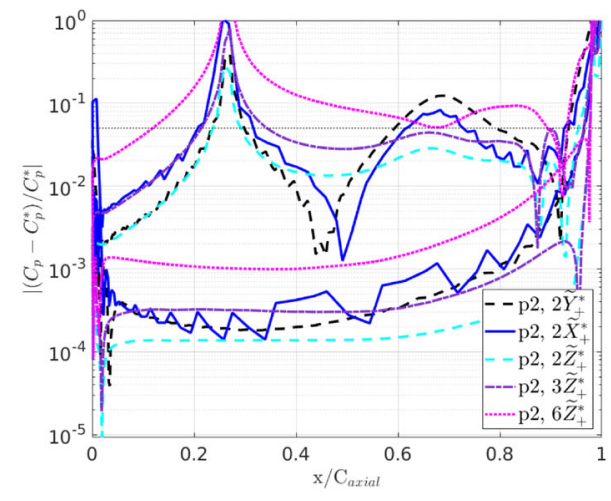

(b)

Fig. 13 Relative absolute differences of the $C_{f s}$ and $C_{p}$ coefficients with respect to the p2 LES simulation on the coarse mesh. Note that the horizontal dotted line marks the $5 \%$ difference threshold. a| $\left|\triangle C_{f s}\right| . \mathbf{b}\left|\triangle C_{p}\right|$ 
The PSD of the pressure signal is also considered in this section to show the effects of these coarse simulations on the pressure spectra in the wake. Figure 14 presents the PSD results of the pressure signal for the considered cases where we can see that indeed the $2 \widetilde{Z}_{+}^{*}$ and $3 \widetilde{Z}_{+}^{*}$ resolutions were able to capture the main peaks very well and follow the $\mathrm{p} 2$ base LES case for high $S t$ numbers. More interestingly these two resolutions were even able to capture the peak value better than the base LES simulation. This may be related also to their accurate representation in the turbulent region near the T.E. as was discussed for the $C_{f s}$ distribution. The $2 \widetilde{X}_{+}^{*}$ and $2 \widetilde{Y}_{+}^{*}$ did not capture any peaks except a spurious one at $S t \approx 5.0$ for the $2 \widetilde{Y}_{+}^{*}$ case. In addition, these two resolutions underestimated the energy content for low $S t$ number whereas the $6 \widetilde{Z}_{+}^{*}$ overestimated the PSD for almost all the captured $S t$ numbers. Based on this study, we can say that the normal as well as streamwise resolutions are more important than the spanwise resolution for this type of flow problems.

For aircraft designers, the mean lift and drag coefficients are very important performance parameters. Therefore, we conduct an additional study concerning the error in the prediction of the $\overline{C_{L}}$ and the $\overline{C_{D}}$ with respect to the DNS results. Table 2 shows the relative errors in addition to the nDOF in each case. From this table we can see that up to $\tilde{Y}_{+}=4 \widetilde{Y}_{+}^{*} \approx 4.0$, one can accurately capture the mean lift and drag coefficients with only a $3.3 \%$ error. In the spanwise direction one can go further up to $\widetilde{Z}_{+}=6 \widetilde{Z}_{+}^{*} \approx 132$ with an error of $3 \%$ and only 80,000 DOF. This is a huge reduction in cost with respect to the p2 base LES simulation of 500,000 DOF. Note that the reduction in $\mathrm{nDOF}$ for the coarse $\widetilde{Y}_{+}$cases was not that large since we only double the first cell height while keeping the rest of the mesh almost the same. In these simulations we were not able to further reduce the resolution in the streamwise $\widetilde{X}_{+}$direction since the mesh is very coarse and a surface conforming high-order mesh was not easily obtained. Moreover, if we doubled the values of $\left(\widetilde{X}_{+}, \widetilde{Y}_{+}, \widetilde{Z}_{+}\right) \approx(40,2,44)$ we still can get a very good solution with only $0.6 \%$ error, using 100,000 DOF. Finally, we show that the combination of all the coarsest resolutions at the same time resulted in a huge error for the mean $\overline{C_{L}}$ coefficient of $\approx 21 \%$.

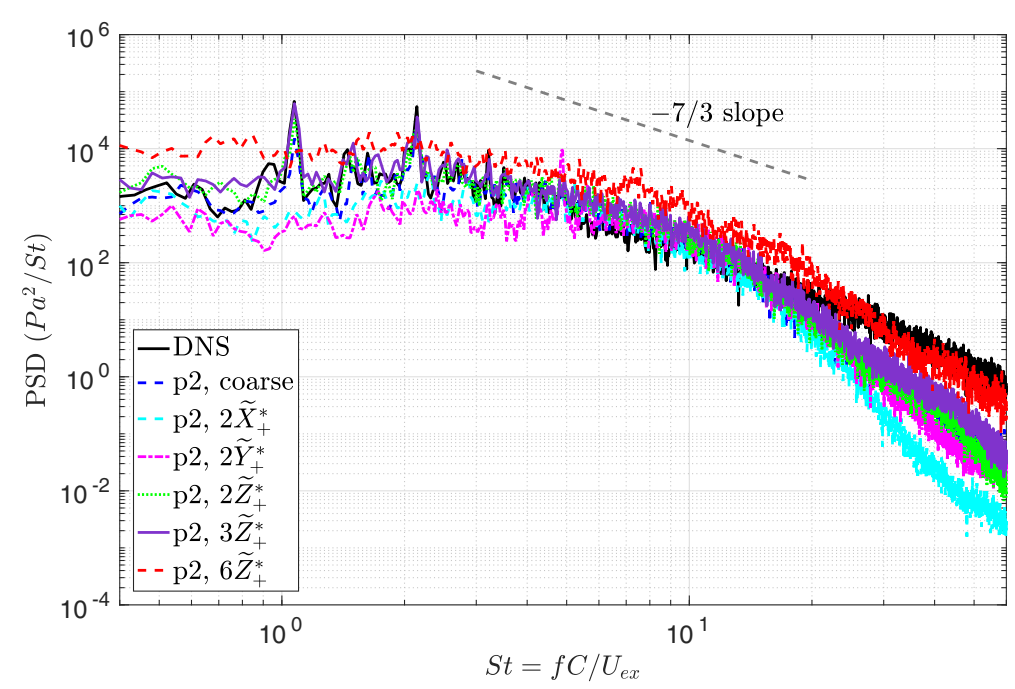

Fig. 14 Comparison of the PSD of pressure at wake point(2) for the coarse LES simulations 
Table 2 Relative error of the mean $\overline{C_{L}}$ and $\overline{C_{D}}$ with respect to the DNS solution for the coarse $\mathbf{L E S}$ simulations

\begin{tabular}{llll}
\hline Cases & $\left(\overline{C_{L}}\right)_{\text {error }} \%$ & $\left(\overline{C_{D}}\right)_{\text {error }} \%$ & $\mathrm{nDOF} \times 10^{5}$ \\
\hline $2 \widetilde{X}_{+}^{*}$ & 0.7 & 0.8 & 2.2 \\
$2 \widetilde{Y}_{+}^{*}$ & 1.2 & 0.9 & 4.6 \\
$4 \widetilde{Y}_{+}^{*}$ & 3.3 & 1.0 & 4.0 \\
$8 \widetilde{Y}_{+}^{*}$ & 11.1 & 20.1 & 3.5 \\
$2 \widetilde{Z}_{+}^{*}$ & 0.0 & 0.2 & 2.5 \\
$3 \widetilde{Z}_{+}^{*}$ & 0.6 & 0.1 & 1.7 \\
$6 \widetilde{Z}_{+}^{*}$ & 3.0 & 0.3 & 0.8 \\
$\left(2 \widetilde{X}_{+}^{*}, 2 \widetilde{Y}_{+}^{*}, 2 \widetilde{Z}_{+}^{*}\right)$ & 0.6 & 0.6 & 1.0 \\
$\left(2 \widetilde{X}_{+}^{*}, 4 \widetilde{Y}_{+}^{*}, 6 \widetilde{Z}_{+}^{*}\right)$ & 20.7 & 6.8 & 0.3 \\
\hline
\end{tabular}

\section{Conclusions}

In this paper, a DNS and LES study for the transitional flow over the T106C blade has been conducted using the high-order FR/CPR method. The DNS solution was established using a 3 ( $4^{\text {th }}$ order) solution on a fine mesh after a systematic h- and p-refinement study. In establishing convergence to the DNS solution, typical mean metrics such as mean lift and drag coefficients as well as the distribution of the skin-friction and isentropic Mach number over the blade, were utilized. Both order and mesh convergence were satisfied for the DNS solution. In addition, the PSD of the pressure and velocity spectra were used to reaffirm the convergence.

Afterwards, a LES resolution study was performed in order to assess the dependence of the mean quantities and the PSD on the values of $\left(\widetilde{X}_{+}, \widetilde{Y}_{+}, \widetilde{Z}_{+}\right)$in wall units, i.e., streamwise, normal, and spanwise directions. It has been found that one can use a mesh with $\left(\widetilde{X}_{+}, \widetilde{Y}_{+}, \widetilde{Z}_{+}\right)=(40,2,40)$ and still get a very good mean lift and drag coefficients with only $0.6 \%$ error. We were also able to show that for coarsening in one direction only, one can still get a good solution with a huge reduction in the nDOF. For instance, the $\widetilde{Z}_{+} \approx$ 132 mesh showed only a $3 \%$ error using 80,000 DOF while keeping $\widetilde{X}_{+} \approx 20, \widetilde{Y}_{+} \approx 1.0$. A similar trend was found for the normal direction with $\tilde{Y}_{+}$up to 4.0.

On the other hand, the pointwise difference in the skin-friction distribution exceeded $5 \%$ in the transition and turbulent regions over the blade surface for all the considered cases. For the PSD, coarsening in the spanwise direction (up to $\widetilde{Z}_{+} \approx 60$ ) had a small effect on the PSD results and was able to capture the peaks accurately.

Based on the results, we conclude that for this type of separation-induced transitional flows in turbomachinery, coarsening in the spanwise direction has a smaller effect on the mean quantities of interest and the PSD in the wake in comparison to the streamwise and normal directions. The results indicate the usefulness of such study in establishing a cost-effective simulation strategy based on a predefined error threshold.

\section{Nomenclature}

$N_{e}$ Number of mesh elements

d Problem dimension

$p$ Polynomial degree for high-order FR/CPR method

$\mathrm{nDOF}$ Number of degrees of freedom

$C$ Blade chord

$U_{\infty}$ Free stream velocity based on the isentropic exit conditions 
$P_{\infty}$ Free stream pressure based on the isentropic exit conditions

$\gamma$ Specific heats ratio of 1.4 for an ideal gas

$M_{i s}$ Isentropic Mach number

$R e_{i s}$ Reynolds number based on the isentropic exit velocity

$P_{2 s}$ Pressure on the blade surface

$C_{p}$ Coefficient of pressure

$C_{f s}$ Coefficient of streamwise friction

$C_{L}$ Lift coefficient

$C_{D}$ Drag coefficient

$\tilde{X}_{+}$Mean mesh resolution in wall units in the blade streamwise direction normalized by $\mathrm{p}+1$

$\tilde{Y}_{+}$Mean mesh resolution in wall units in the blade normal direction normalized by $\mathrm{p}+1$

$\widetilde{Z}_{+}$Mean mesh resolution in wall units in the spanwise direction normalized by $\mathrm{p}+1$

$T_{c}$ Characteristic time

$f$ Frequency in $\mathrm{Hz}$

St Strouhal number

$\hat{G}$ Fourier transformed amplitude of the one-sided spectrum for a time signal $G$

PSD Power spectral density

FR/CPR Flux reconstruction or correction procedure via reconstruction method

LES Large eddy simulations

DNS Direct numerical simulations

RANS Reynolds averaged Navier-Stokes equations

\section{Acknowledgements}

The authors would like to acknowledge the financial support by AFOSR under grant FA9550-16-1-0128 and US Army Research Office under grant W911NF-15-1-0505.

Authors' contributions

The idea was suggested by the corresponding author, and the first author carried out the numerical simulations, coding and necessary data analysis. Both authors have read and approved the manuscript.

Funding

AFOSR grant (FA9550-16-1-0128), US Army Research Office grant (W91 1NF-15-1-0505).

Availability of data and materials

All data generated or analyzed during this study are included in this published article. Parts of the data and materials are available upon request.

\section{Competing interests}

The authors declare that they have no competing interests.

Received: 1 October 2019 Accepted: 13 November 2019

Published online: 28 December 2019

\section{References}

1. Smagorinsky J (1963) General circulation experiments with the primitive equations. Mon Wea Rev 91(3):99-164

2. Gourdain N, Sicot F, Duchaine F, Gicquel L (2014) Large eddy simulation of flows in industrial compressors: a path from 2015 to 2035. Philos Trans R Soc A Math Phys Eng Sci 372(2022):20130323

3. Tyacke JC, Tucker PG (2015) Future Use of Large Eddy Simulation in Aero-engines. J Turbomach 137(8). https://doi. org/10.1115/1.4029363

4. Wang ZJ, Li Y, Jia F, Laskowski GM, Kopriva J, Paliath U, Bhaskaran R (2017) Towards industrial large eddy simulation using the FR/CPR method. Comput Fluids 156:579-589

5. Bhaskaran R, Jia F, Laskowski GM, Wang ZJ, Paliath U (2017) Towards High-Order Large Eddy Simulation of Aero-Thermal Flows for Turbomachinery Applications. In: ASME Turbo Expo 2017, Charlotte. pp 02-41011. https:// doi.org/10.1115/gt2017-63358 
6. Sandberg RD, Michelassi V (2019) The Current State of High-Fidelity Simulations for Main Gas Path Turbomachinery Components and Their Industrial Impact. Flow Turbul Combust 102(4):797-848

7. Li X-L, Fu D-X, Ma Y-W, Liang X (2010) Direct numerical simulation of compressible turbulent flows. Acta Mech Sin 26(6):795-806

8. Slotnick J, Khodadoust A, Alonso J, Darmofal D, Gropp W, Lurie E, Mavriplis D CFD Vision 2030 Study: A Path to Revolutionary Computational Aerosciences. Technical Report NASA/CR-2014-218178. NASA Langley Research Center

9. Tucker PG (2013) Trends in turbomachinery turbulence treatments. Progress Aerosp Sci 63:1-32

10. Tyacke J, Vadlamani NR, Trojak W, Watson R, Ma Y, Tucker PG (2019) Turbomachinery simulation challenges and the future. Progress Aerosp Sci:100554. https://doi.org/10.1016/j.paerosci.2019.100554

11. Laskowski GM, Kopriva J, Michelassi V, Shankaran S, Paliath U, Bhaskaran R, Wang Q, Talnikar C, Wang ZJ, Jia F (2016) Future Directions of High Fidelity CFD for Aerothermal Turbomachinery Analysis and Design. In: 46th AIAA Fluid Dynamics Conference. AIAA AVIATION Forum. American Institute of Aeronautics and Astronautics. https://doi.org/ 10.2514/6.2016-3322

12. Hillewaert K, Carton de Wiart C, Verheylewegen G, Arts T (2014) Assessment of a High-Order Discontinuous Galerkin Method for the Direct Numerical Simulation of Transition at Low-Reynolds Number in the T106c High-Lift Low Pressure Turbine Cascade. In: ASME Turbo Expo 2014, Dusseldorf. pp 2014-26739. https://doi.org/10.1115/gt201426739

13. Marty J (2014) Numerical investigations of separation-induced transition on high-lift low-pressure turbine using RANS and LES methods. Proc Inst Mech Eng Part J Power Energy 228(8):924-952

14. Sandberg RD, Michelassi V, Pichler R, Chen L, Johnstone R (2015) Compressible Direct Numerical Simulation of Low-Pressure Turbines_Part I: Methodology. J Turbomach 137(5):051011-05101110

15. Wheeler APS, Sandberg RD, Sandham ND, Pichler R, Michelassi V, Laskowski G (2016) Direct Numerical Simulations of a High-Pressure Turbine Vane. J Turbomach 138(7):071003-0710039

16. Garai A, Diosady LT, Murman SM, Madavan NK (2016) DNS of Low-Pressure Turbine Cascade Flows With Elevated Inflow Turbulence Using a Discontinuous-Galerkin Spectral-Element Method. In: ASME Turbo Expo 2016. American Society of Mechanical Engineers. pp 2016-56700. https://doi.org/10.1115/gt2016-56700

17. Pichler R, Sandberg RD, Michelassi V (2016) Assessment of Grid Resolution Requirements for Accurate Simulation of Disparate Scales of Turbulent Flow in Low-Pressure Turbines. In: ASME Turbo Expo 2016, Seoul. pp 02-39030. https://doi.org/10.1115/gt2016-56858

18. Cassinelli A, Montomoli F, Adami P, Sherwin SJ (2018) High Fidelity Spectral/hp Element Methods for Turbomachinery. In: ASME Turbo Expo 2018, Oslo. pp 02-42020. https://doi.org/10.1115/gt2018-75733

19. Michelassi V, Chen L-W, Pichler R, Sandberg RD (2015) Compressible Direct Numerical Simulation of Low-Pressure Turbines_Part II: Effect of Inflow Disturbances. J Turbomach 137(7):071005-07100512

20. Rai M (2011) A Direct Numerical Simulation of Flow Through a Low Pressure Turbine Stage. In: 41st AIAA Fluid Dynamics Conference And Exhibit. Fluid Dynamics and Co-located Conferences. American Institute of Aeronautics and Astronautics. https://doi.org/10.2514/mfd11

21. Hu S, Zhou C, Xia Z, Chen S (2017) Large Eddy Simulation and CDNS Investigation of T106c Low-Pressure Turbine. J Fluids Eng 140(1):011108-01110812

22. Wang ZJ (2007) High-order methods for the Euler and Navier-Stokes equations on unstructured grids. Prog Aerosp Sci 43(1):1-41

23. Wang ZJ, Fidkowski K, Abgrall R, Bassi F, Caraeni D, Cary A, Deconinck H, Hartmann R, Hillewaert K, Huynh Ht, Kroll N, May G, Persson P-O, van Leer B, Visbal M (2013) High-order CFD methods: current status and perspective. Int J Numer Meth Fluids 72(8):811-845

24. Huynh HT (2007) A Flux Reconstruction Approach to High-Order Schemes Including Discontinuous Galerkin Methods. In: 18th AIAA Computational Fluid Dynamics Conference. American Institute of Aeronautics and Astronautics, AIAA 2007-4079. https://doi.org/10.2514/6.2007-4079

25. Wang ZJ, Gao H (2009) A Unifying Lifting Collocation penalty formulation including the discontinuous Galerkin, spectral volume/difference methods for conservation laws on mixed grids. J Comput Phys 228(21):8161-8186

26. Wang ZJ, Gao H, Haga T A unifying discontinuous formulation for hybrid meshes. In: Adaptive High-order Methods in Computational Fluid Dynamics. World Scientific. pp 423-453. https://doi.org/10.1142/9789814313193_0015

27. Liu Y, Vinokur M, Wang ZJ (2006) Spectral difference method for unstructured grids I: Basic formulation. J Comput Phys 216(2):780-801

28. Wang ZJ (2002) Spectral (Finite) Volume Method for Conservation Laws on Unstructured Grids, Basic Formulation: Basic Formulation. J Comput Phys 178(1):210-251

29. Reed WH, Hill TR Triangular Mesh Methods for the Neutron Transport Equation. Technical Report LA-UR-73-479; CONF-730414-2, Los Alamos Scientific Lab., N.Mex. (USA)

30. Discontinuous Galerkin Methods - Theory, Computation and Applications(Cockburn B, Karniadakis GE, Shu C-W, eds.). Springer. https://doi.org/10.1007/978-3-642-59721-3

31. Hesthaven JS, Warburton T Nodal Discontinuous Galerkin Methods: Algorithms, Analysis, and Applications. 1st edn.. Springer. https://doi.org/10.1007/978-0-387-72067-8

32. Zhang L, Wei L, Lixin H, Xiaogang D, Hanxin Z (2012) A class of hybrid DG/FV methods for conservation laws I: Basic formulation and one-dimensional systems. J Comput Phys 231(4):1081-1103

33. Visbal MR, Rizzetta DP (2002) Large-Eddy Simulation on Curvilinear Grids Using Compact Differencing and Filtering Schemes. J Fluids Eng 124(4):836

34. Kawai S, Shankar SK, Lele SK (2010) Assessment of localized artificial diffusivity scheme for large-eddy simulation of compressible turbulent flows. J Comput Phys 229(5):1739-1762

35. Bogey C, Bailly C (2006) Large eddy simulations of round free jets using explicit filtering with/without dynamic Smagorinsky model. Int J Heat Fluid Flow 27(4):603-610

36. Garmann DJ, Visbal MR, Orkwis PD (2013) Comparative study of implicit and subgrid-scale model large-eddy simulation techniques for low-Reynolds number airfoil applications. Int J Numer Methods Fluids 71(12):1546-1565 
37. Uranga A, Persson P-O, Drela M, Peraire J (2011) Implicit Large Eddy Simulation of transition to turbulence at low Reynolds numbers using a Discontinuous Galerkin method. Int J Numer Meth Engng 87(1-5):232-261

38. Vermeire BC, Nadarajah S, Tucker PG (2016) Implicit large eddy simulation using the high-order correction procedure via reconstruction scheme. Int J Numer Meth Fluids 82(5):231-260

39. Zhu H, Fu S, Shi L, Wang ZJ (2016) Implicit Large-Eddy Simulation for the High-Order Flux Reconstruction Method. AIAA J 54(9):2721-2733

40. Alhawwary M, Wang ZJ (2018) Fourier analysis and evaluation of DG, FD and compact difference methods for conservation laws. J Comput Phys 373:835-862

41. Alhawwary MA, Wang ZJ (2018) Comparative Fourier Analysis of DG, FD and Compact Difference schemes. In: 2018 Fluid Dynamics Conference, AIAA Aviation Forum. American Institute of Aeronautics and Astronautics, AIAA-2018-4267, Atlanta

42. 4th International Workshop on High-Order CFD Methods. https://how4.cenaero.be/. Accessed 1 Oct 2019

43. Michálek J, Monaldi M, Arts T (2012) Aerodynamic Performance of a Very High Lift Low Pressure Turbine Airfoil (T106c) at Low Reynolds and High Mach Number With Effect of Free Stream Turbulence Intensity. J Turbomach 134(6):061009-06100910

44. Huynh HT (2009) A Reconstruction Approach to High-Order Schemnes Including Discontinuous Galerkin for Diffusion. In: 47th AIAA Aerospace Sciences Meeting Including The New Horizons Forum and Aerospace Exposition. American Institute of Aeronautics and Astronautics. pp 2009-403. https://doi.org/10.2514/6.2009-403

45. Vincent PE, Castonguay P, Jameson A (2011) A New Class of High-Order Energy Stable Flux Reconstruction Schemes, J Sci Comput 47(1):50-72

46. Gao H, Wang ZJ, Huynh HT (2013) Differential Formulation of Discontinuous Galerkin and Related Methods for the Navier-Stokes Equations. Commun Comput Phys 13(04):1013-1044

47. Huynh HT, Wang ZJ, Vincent PE (2014) High-order methods for computational fluid dynamics: A brief review of compact differential formulations on unstructured grids. Comput Fluids 98:209-220

48. Yu M, Wang ZJ, Liu Y (2014) On the accuracy and efficiency of discontinuous Galerkin, spectral difference and correction procedure via reconstruction methods. J Comput Phys 259:70-95

49. Wang ZJ (2016) A perspective on high-order methods in computational fluid dynamics. Sci China Phys Mech Astron 59(1):614701

50. Wang ZJ, Huynh HT (2016) A review of flux reconstruction or correction procedure via reconstruction method for the Navier-Stokes equations. Mech Eng Rev 3(1):15-004751500475

51. Bassi F, Rebay S, Mariotti G, Pedinotti S, Savini M (1997) A Higher-order accurate discontinuous Finite Element Method for inviscid and viscous turbomachinery flows. In: Decuypere R, Dibelius G (eds). Proceedings of 2nd European Conference on Turbomachinery-Fluid Dynamics and Thermodynamics. Antwerp: Technologische Instituut, Antwerpen. pp 99-108

52. Chen RF, Wang ZJ (2000) Fast, Block Lower-Upper Symmetric Gauss-Seidel Scheme for Arbitrary Grids. AIAA J 38(12):2238-2245

53. Yoon S, Jameson A (1988) Lower-upper Symmetric-Gauss-Seidel method for the Euler and Navier-Stokes equations. AIAA J 26(9):1025-1026

54. Pacciani R, Marconcini M, Fadai-Ghotbi A, Lardeau S, Leschziner MA (2009) Calculations of High-Lift Cascades in Low Pressure Turbine Conditions Using a Three-Equation Model. In: ASME Turbo Expo 2009. pp 433-442. https://doi.org/ 10.1115/gt2009-59557

55. Benyahia A, Castillon L, Houdeville R (2011) Prediction of Separation-Induced Transition on High Lift Low Pressure Turbine Blade. In: ASME Turbo Expo 2011. pp 1835-1846. https://doi.org/10.1115/gt2011-45566

56. Pacciani R, Marconcini M, Arnone A, Bertini F (2013) Predicting High-Lift Low-Pressure Turbine Cascades Flow Using Transition-Sensitive Turbulence Closures. J Turbomach 136(5):051007-05100711

57. Welch P (1967) The use of fast Fourier transform for the estimation of power spectra: A method based on time averaging over short, modified periodograms. IEEE Trans Audio Electroacoustics 15(2):70-73

58. Alhawwary MA A C++ toolbox for computing Discrete and Fast Fourier Transforms (DFT,FFT), Power Spectral Density (PSD) estimates, and the sound pressure level (SPL) in (dB). https://github.com/mhawwary/FFTpsd. Accessed 1 Oct 2019

59. Kolmogorov AN (1962) A refinement of previous hypotheses concerning the local structure of turbulence in a viscous incompressible fluid at high Reynolds number. J Fluid Mech 13(1):82-85

60. George WK, Beuther PD, Arndt REA (1984) Pressure spectra in turbulent free shear flows. J Fluid Mech 148:155-191

61. Jones BG, Adrian RJ, Nithianandan CK, Jr HPP (1979) Spectra of Turbulent Static Pressure Fluctuations in Jet Mixing Layers. AIAA J. https://doi.org/10.2514/6.1977-1370

62. Nelkin M (1994) Universality and scaling in fully developed turbulence. Adv Phys 43(2):143-181

\section{Publisher's Note}

Springer Nature remains neutral with regard to jurisdictional claims in published maps and institutional affiliations. 